\title{
THE BRAUER GROUP IS TORSION
}

\author{
DAVID J. SALTMAN
}

\begin{abstract}
We present a new proof that if $A$ is an Azumaya algebra over a commutative ring $R$ of rank $n^{2}$, then $A^{n}=A \otimes_{R} \cdots \otimes_{R} A$ is a split Azumaya algebra $\operatorname{End}_{R}(P)$. We provide a description of $P$, including that it is a direct summand of $A^{n}$.
\end{abstract}

In [3], a well-known fact about the Brauer group of a field was generalized to show that the Brauer group of a commutative ring was torsion. In fact, if $A$ is Azumaya of constant rank $n^{2}$ over $R$, then $A^{n}\left(=A \otimes_{R} \cdots \otimes_{R} A\right) \simeq \operatorname{End}_{R}(P)$ for $P$ an progenerator. In this note we will present a relatively elementary proof of this theorem, and we will also describe $P$ as a specific direct summand of $A^{n}$. It should be noted that our argument is related to one that has been used in the case $R$ is a field (see, for example, [6]).

Our proof begins much like the standard one, with faithfully flat splittings. Fix $A$ and $R$ as above. There is a faithfully flat commutative ring extension $R \subset S$ such that $A \otimes_{R} S \cong M_{n}(S) \cong \operatorname{End}_{S}(V)$ where $V$ is a free $S$ module of rank $n$ (e.g. [4, p. 106]). We call such an $S$ and $V$ a free splitting of $A$. Given such a free splitting, we will identify $A \otimes_{R} S, M_{n}(S)$ and $\operatorname{End}_{S}(V)$. Also, if $r \geqslant 1$ is an integer, then $A^{r} \otimes_{R} S$ is naturally isomorphic to $\operatorname{End}_{S}\left(V^{r}\right)=\operatorname{End}_{S}\left(V \otimes_{S} \cdots \otimes_{S} V\right)$. Identify $A^{r} \otimes_{R} S$ with this endomorphism ring.

Let $\operatorname{tr}: A \rightarrow R$ be the reduced trace map. In [4, p. 112] (result attributed to Goldman), the reduced trace map is used to define a very useful element $\alpha \in A$ $\otimes_{R} A$. In fact, $\alpha$ is uniquely defined by the property that $\alpha=\sum x_{i} \otimes y_{i}$ where $\operatorname{tr}(a)=\Sigma x_{i} a y_{i}$ for all $a \in A$. The properties of $\alpha$ are listed in the next lemma.

LEMMA 1. (a) $\alpha^{2}=1$.

(b) $\alpha(a \otimes b)=(b \otimes a) \alpha$ for all $a, b \in A$.

(c) Let $S, V$ be any free splitting of $A$. Consider

$$
\alpha \otimes 1 \in A^{2} \otimes_{R} S=\operatorname{End}_{S}(V \otimes V) .
$$

Then $\alpha \otimes 1$ is the map defined by $\alpha \otimes 1(v \otimes w)=w \otimes v$.

Proof. Parts (a) and (b) are directly from [4]. As for (c), the uniqueness of $\alpha$ is used to show that $\alpha \otimes 1=\Sigma_{i, j} e_{i j} \otimes e_{j i}$, where the $e_{i j}$ are any matrix units for $A \otimes_{R} S$. Translated into maps, that is just (c).

Received by the editors April 28, 1980.

AMS (MOS) subject classifications (1970). Primary 16A16; Secondary 13A20.

Key words and phrases. Azumaya algebra, Brauer group. 
The above facts can be generalized to higher tensor powers of $A$ as follows. Let $m$ be an integer $m \leqslant n$. The symmetric group, $S_{m}$, acts on $A^{m}=A \otimes_{R} \cdots \otimes_{R} A$ in the natural way. If $S$ and $V$ are a free splitting of $A, A^{m} \otimes_{R} S$ has been identified with $\operatorname{End}_{S}\left(V^{m}\right)$. The natural action of $S_{m}$ on $V^{m}$ induces the action of $S_{m}$ on $A^{m} \otimes_{R} S$.

THEOREM 2. For each $\sigma \in S_{m}$ there is a unit $\alpha_{\sigma} \in A^{m}$ such that

(a) $\alpha_{\sigma}^{-1} \beta \alpha_{\sigma}=\sigma(\beta)$ for all $\beta \in A^{m}$.

(b) The map $\sigma \rightarrow \alpha_{\sigma}$ is a homomorphism from $S_{m}$ to the group of units of $A^{m}$.

(c) If $S$ and $V$ are a free splitting of $A$, then $\alpha_{\sigma} \otimes 1 \in A^{m} \otimes_{R} S$, considered as an endomorphism of $V^{m}$, is just the map $\sigma$.

(d) The $\alpha_{\sigma}$ 's are linearly independent over $R$.

Proof. For a fixed $S$ and $V$, we use (c) to define $\alpha_{\sigma}^{\prime} \in A^{m} \otimes_{R} S$. To prove parts (a) and (b), it suffices to show that the $\alpha_{\sigma}^{\prime}$ are in the image of $A^{m}$. As $S_{m}$ is generated by 2-cycles, we may assume $\sigma$ is a 2-cycle. But after obvious identifications, this case is covered by Lemma 1 . Part (c) for arbitrary $S$ and $V$ follows from 1(c). As for (d), it suffices to prove that the $\alpha_{\sigma} \otimes 1$ 's are linearly independent over $S$, and this follows from the easy observation that $V^{m}$ is a faithful module over the group algebra $S\left(S_{m}\right)$. Q.E.D.

We now turn to the algebra $A^{n}$, where we recall that $A$ has rank $n^{2}$ over $R$. In $A^{n}$, we define $\beta=\Sigma_{S_{n}} \operatorname{sgn}(\sigma) \alpha_{\sigma}$. Of course, $\operatorname{sgn}(\sigma)$ is \pm 1 depending on whether the permutation $\sigma$ is even or odd. Let $S$ and $V$ be any free splitting of $A$. Consider $\beta \otimes 1$ as an $S$ endomorphism of $V^{n} .(\beta \otimes 1)\left(x_{1} \otimes \cdots \otimes x_{n}\right)=0$ if $x_{i}=x_{j}$ for $i \neq j$. If $v_{1}, \ldots, v_{n}$ are an $S$ basis of $V$, we quickly see that $(\beta \otimes 1)\left(V^{n}\right)$ is generated over $S$ by the single element $w=\Sigma_{S_{n}} \operatorname{sgn}(\sigma) v_{\sigma(1)} \otimes \cdots \otimes v_{\sigma(n)}$. What is more, $w$ is part of a free basis of $V^{n}$. Let $W$ be the kernel of $\beta \otimes 1$, so $W$ is a direct summand of $V^{n}$. The left ideal $\left(A^{n} \otimes_{R} S\right)(\beta \otimes 1)$, as a subset of $\operatorname{End}_{S}\left(V^{n}\right)$, is exactly those endomorphisms which are zero on $W$. Now set $J=A^{n} \beta$. Then $\left(A^{n} / J\right) \otimes_{R} S \cong\left(A^{n} \otimes_{R} S\right) /\left(A^{n} \otimes_{R} S\right)(\beta \otimes 1) \simeq \operatorname{Hom}_{S}\left(W, V^{n}\right)$ as $S$ modules, so $\left(A^{n} / J\right) \otimes_{R} S$ is an $S$ progenerator. Since $S$ is faithfully flat over $R, A^{n} / J$ is an $R$ progenerator (e.g. [1, p. 34]). Since $A^{n}$ is Azumaya over $R \cdot A^{n} / J$ is a projective $A^{n}$ module and so $J$ is an $A^{n}$ direct summand. We have proved most of the first part of the following theorem.

TheOREM 3. (a) $J=A^{n} \beta$ is a $A^{n}$-direct summand of $A^{n}$, and an $R$ progenerator of rank $n^{n}$.

(b) $A^{n} \cong \operatorname{End}_{R}(J)$.

Proof. Part (a) has been shown, except for the trivial calculation of the rank of $J$. As for (b), $J$ is a faithful $A^{n}$ module because it is a faithful $R$ module (e.g. [2, p. 54]). The injection $A^{n} \rightarrow \operatorname{End}_{R}(J)$ must be surjective using the double centralizer theorem and the equal $R$ ranks of $A^{n}$ and $\operatorname{End}_{R}(J)$ (e.g. [2, p. 57]). Q.E.D.

As a final remark let us note that the proof of Theorem 3 is a special case of more general phenomenon. If $\boldsymbol{B}$ is an Azumaya algebra over a field $\boldsymbol{F}$ of dimension $n^{2}$, the rank of any $b \in B$ can be unambiguously defined as $(1 / n)\left(\operatorname{dim}_{F} B b\right)$. Let $A$ 
be an Azumaya algebra over $R$, with rank $n^{2}$, and let $\mathfrak{B} \subseteq R$ be a prime ideal of $R$. For any $a \in A$, we define the rank of $a$ at $\mathfrak{P}$ to be the rank of $a \otimes 1 \in A \otimes_{R} K$, where $K$ is the field of quotients of $R / \mathfrak{B}$. We say $a \in A$ has constant rank $r$ if $a$ has rank $r$ at every prime ideal of $R$. Using arguments as in [5, p. 339], one can show that if $a$ has constant rank $r$ then $A a$ is an $R$ progenerator of rank $n r$ and an $A$ direct summand of $A$. The element $\beta$ used above has constant rank one.

\section{BIBLIOGRAPHY}

1. Nathan Bourbaki, Commutative algebra, Addison-Wesley, Reading, Mass., 1972.

2. F. DeMeyer and E. Ingraham, Separable algebras over commutative rings, Lecture Notes in Math., vol. 181, Springer-Verlag, Berlin and New York, 1971.

3. A. Grothendieck, Le groupe de Brauer. I, Dix Exposés sur la Cohomologie des Schémas, North-Holland, Amsterdam; Masson, Paris, 1968, pp. 46-66.

4. M. A. Knus and M. Ojanguren, Théorie de la descente et algèbre d'Azumaya, Lecture Notes in Math., vol. 389, Springer-Verlag, Berlin and New York, 1974.

5. D. Saltman, Norm polynomials and algebras, J. Algebra 62 (1980), 332-345.

6. T. Tamagawa, Representation theory and the notion of the discriminant, Algebraic Number Theory, Japan Society for the Promotion of Science, Tokyo, 1977.

Departiment of Mathematics, Yale University, New Haven, Connecticut 06520 